This is an electronic reprint of the original article. This reprint may differ from the original in pagination and typographic detail.

\author{
Author(s): Sorri, Juha; Greenlees, Paul; Papadakis, Philippos; Konki, Joonas; Cox, Daniel; Auranen, \\ Kalle; Partanen, Jari; Sandzelius, Mikael; Pakarinen, Janne; Rahkila, Panu; Uusitalo, \\ Juha; Herzberg, R.-D.; Smallcombe, J.; Davies, P.J.; Barton, C.J.; Jenkins, D.G.
}

Title: Determination of absolute internal conversion coefficients using the SAGE spectrometer

Year: $\quad 2016$

Version:

Please cite the original version:

Sorri, J., Greenlees, P., Papadakis, P., Konki, J., Cox, D., Auranen, K., Partanen, J., Sandzelius, M., Pakarinen, J., Rahkila, P., Uusitalo, J., Herzberg, R.-D., Smallcombe, J., Davies, P.J., Barton, C.J., \& Jenkins, D.G. (2016). Determination of absolute internal conversion coefficients using the SAGE spectrometer. Nuclear Instruments and Methods in Physics Research Section A: Accelerators, Spectrometers, Detectors and Associated Equipment, 812, 24-32. https://doi.org/10.1016/j.nima.2015.12.041

All material supplied via JYX is protected by copyright and other intellectual property rights, and duplication or sale of all or part of any of the repository collections is not permitted, except that material may be duplicated by you for your research use or educational purposes in electronic or print form. You must obtain permission for any other use. Electronic or print copies may not be offered, whether for sale or otherwise to anyone who is not an authorised user. 


\title{
Determination of absolute internal conversion coefficients using the SAGE spectrometer
}

\author{
J. Sorria,*, P. T. Greenlees ${ }^{\mathrm{a}}$, P. Papadakis ${ }^{\mathrm{a}}$, J. Konki ${ }^{\mathrm{a}}$, D. M. Cox ${ }^{\mathrm{a}, \mathrm{b}}$, \\ K. Auranen ${ }^{\mathrm{a}}$, J. Partanen ${ }^{\mathrm{a}}$, M. Sandzelius ${ }^{\mathrm{a}}$, J. Pakarinen ${ }^{\mathrm{a}}$, P. Rahkila ${ }^{\mathrm{a}}$,

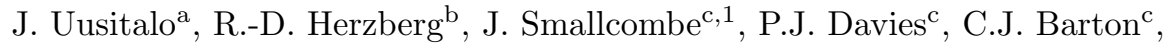 \\ D.G. Jenkins ${ }^{\mathrm{c}}$ \\ ${ }^{a}$ University of Jyvaskyla, Department of Physics, P.O. Box 35, FI-40014 University of \\ Jyvaskyla, Finland \\ ${ }^{b}$ Department of Physics, University of Liverpool, Oxford Street, Liverpool, L69 7ZE, U.K. \\ ${ }^{c}$ Department of Physics, University of York, Heslington, York YO10 5DD, United Kingdom
}

\begin{abstract}
A non-reference based method to determine internal conversion coefficients using the SAGE spectrometer is carried out for transitions in the nuclei ${ }^{154} \mathrm{Sm},{ }^{152} \mathrm{Sm}$ and ${ }^{166} \mathrm{Yb}$. The Normalised-Peak-to-Gamma method is in general an efficient tool to extract internal conversion coefficients. However, in many cases the required well-known reference transitions are not available. The data analysis steps required to determine absolute internal conversion coefficients with the SAGE spectrometer are presented. In addition, several background suppression methods are introduced and an example of how ancillary detectors can be used to select specific reaction products is given. The results obtained for ground-state band E2 transitions show that the absolute internal conversion coefficients can be extracted using the methods described with a reasonable accuracy. In some cases of less intense transitions only an upper limit for the internal conversion coefficient could be given.
\end{abstract}

Keywords: electron spectroscopy, background subtraction, energy reconstruction, internal conversion coefficient, silicon detector

\footnotetext{
* Corresponding author

Email address: juha.m.t.sorri@jyu.fi (J. Sorri)

${ }^{1}$ Current address: TRIUMF, 4004 Wesbrook Mall, Vancouver, BC V6T 2A3, Canada
} 


\section{Introduction}

2 The analysis methods described in this paper have been developed primarily 3 for use with the SAGE (Silicon And GErmanium) spectrometer located at the 4 Accelerator Laboratory of the University of Jyväskylä (JYFL). The spectrom5 eter was constructed with the aim of performing simultaneous in-beam gamma 6 ray and internal conversion electron spectroscopic studies by combining the 7 JUROGAMII germanium array [1] with an electron spectrometer. Detailed dis8 cussions of the construction and performance of the SAGE spectrometer can be 9 found in Refs. [2-4]. Further combination of the SAGE spectrometer with the RITU [5, 6] gas-filled recoil separator and the GREAT [7] focal plane spectrometer allows the use of the recoil-decay tagging technique [8-10]. The primary beam from the JYFL K-130 cyclotron is used to induce nuclear reactions at the target. Depending on the experiment, the reaction products enter RITU and are either dumped or transported to the GREAT spectrometer at the focal plane. The prompt $\gamma$-rays emitted in the de-excitation of the populated nuclei are detected with the germanium detectors of the JUROGAMII array and conversion electrons are transported upstream from the target position by an electromagnetic solenoid and detected with a segmented silicon detector. The electrons travel along a helical path following the magnetic field lines in the solenoid, the radius of which is dependent the electron velocity perpendicular to the magnetic field and the magnetic field strength. A high voltage barrier is used to reduce the extremely high flux of $\delta$-electrons produced by interactions of the ion beam with the atomic electrons of the target material. The silicon detector segments have small average size, which has a drawback at high electron energies. The electron interaction volume grows large and many of the detected electrons deposit energy in more than one segment. An algorithm designed to reduce background generated by scattering between segments is discussed later in the paper. In addition, the fact that the radius of the helical path followed by the electrons is energy dependent provides an opportunity to perform further background filtering. An algorithm exploiting these properties is also presented. 


\section{Experiment details}

The nuclear structure properties of ${ }^{154} \mathrm{Sm}$ were recently discussed by Smallcombe et al. [11]. The main goal of the experiment (here referred to as S06) was to determine internal conversion coefficients from the excited rotational bands of ${ }^{154} \mathrm{Sm}$ in order to test the hypothesis that the bands have vibrational ( $\beta$-band) structure. Coulomb excitation was used to populate the excited energy levels in ${ }^{154} \mathrm{Sm}$ by using an enriched target of ${ }^{154} \mathrm{Sm}$ which was irradiated with a beam of ${ }^{16} \mathrm{O}$. Subsequent to the experiment S06, an additional test experiment ST1 was performed. For the ST1 test run the pre-amplifier signals of the outer segments of the SAGE Si-detector were fed through voltage dividers that increased the maximum detectable energy up to $30-40 \mathrm{MeV}$ from the original 2-2.5 MeV. The reasons for this modification and discussion of the results obtained are presented later in this manuscript. The various parameters related to S06 and ST1 experiments are summarised in table 1. In the aforementioned work by Smallcombe et al., the analysis of the electron-gamma coincidence data and extraction of the internal conversion coefficients relies on the Normalised-Peak-to-Gamma (NPG) method [12]. The NPG method is based on the observation of known transitions and therefore can not be applied to cases where these reference points are missing. A standalone procedure to determine internal conversion coefficients (ICCs) from in-beam data along with methods to reduce the underlying electron background are introduced in this paper. The discussion relies on the same dataset (S06) as that used by Smallcombe et al. and the data-analysis parameters such as trigger conditions, event widths etc. are kept as close as possible to those introduced in Ref. [11] in order to enable direct comparison between results. Partial level schemes of studied nuclei are shown in figure 1.

\subsection{Determination of the gamma-ray and electron detection efficiencies}

In order to reduce the total counting rate, it is usual that $\mathrm{Sn}(0.1 \mathrm{~mm})$ and $\mathrm{Cu}(0.5 \mathrm{~mm})$ absorbers are placed between the target and the germanium detectors of JUROGAMII. In S06 experiment, the absorbers were removed in 
Table 1: Summary of various parameters used in the two experiments presented in this work. $\mathrm{U}_{H V}$ is the voltage applied to $\mathrm{HV}$ barrier and $\mathrm{I}_{\text {coils }}$ is the current through the SAGE magnetic coils.

\begin{tabular}{lllllll}
\hline & \multicolumn{2}{c}{ Target } & \multicolumn{2}{c}{ Beam } & \multicolumn{2}{c}{ SAGE } \\
Run & ElementThickness & Ion & Energy & $\mathrm{U}_{H V}$ & $\mathrm{I}_{\text {coils }}$ \\
& & {$\left[\mathrm{mg} / \mathrm{cm}^{2}\right]($ enrich. $)$} & {$[\mathrm{MeV}]$} & {$[\mathrm{kV}]$} & {$[\mathrm{A}]$} \\
\hline \hline \multirow{2}{*}{ S06 } & ${ }^{154} \mathrm{Sm}$ & $1.5(99 \%)$ & ${ }^{16} \mathrm{O}$ & 65 & -20 & 800 \\
\hline \multirow{2}{*}{ ST1 } & ${ }^{154} \mathrm{Sm}$ & $1.1(98.69 \%)$ & ${ }^{16} \mathrm{O}$ & $35 / 65$ & -20 & 800 \\
& ${ }^{154} \mathrm{Gd}$ & $4.16(80 \lesssim \%)^{a}$ & ${ }^{16} \mathrm{O}$ & 65 & -20 & 0 \\
\hline
\end{tabular}

${ }^{a}$ Estimated from $\gamma$-ray spectrum.

order to improve the detection of Sm X-rays. The absolute gamma-ray detection efficiency was measured using calibrated ${ }^{133} \mathrm{Ba},{ }^{152} \mathrm{Eu}$ and ${ }^{207} \mathrm{Bi}$ sources. The resulting $\gamma$-ray detection efficiency curve is presented in figure 2 . Note that the curve deviates from the one shown in Ref. [4] as S06 was run without absorber foils of the JUROGAMII array. For both the SAGE $\gamma$-ray and conversion electron efficiency curves the data are fitted with a function of the form

$$
\epsilon(E)=\operatorname{Exp}\left[\sum_{i=0}^{n} a_{i} \times \ln \left(\frac{E}{E_{0}}\right)\right]
$$

where $a_{i}$ and $\mathrm{E}_{0}$ are fitted coefficients and $\mathrm{E}$ is the energy of the $\gamma$-ray or internal conversion electron. The electron detection efficiency was determined by using calibrated open ${ }^{133} \mathrm{Ba}$ and ${ }^{207} \mathrm{Bi}$ conversion electron sources. The resulting electron detection efficiency curve is shown in figure 3 . It can be seen from the figure 3 that it is possible to improve the efficiency for detection of higher energy electrons by using an add-back procedure. Details of this procedure are discussed later in the text. The calibration runs during the experiment were made with no voltage applied to the HV barrier. The effect of the barrier shown in detail in figure 4 is an average behaviour deduced by fitting data obtained from several measurements carried out subsequent to the S06 experiment. As can be seen from figure 4 , the effect of the $\mathrm{HV}$ barrier is negligible for electron energies over $200 \mathrm{keV}$, even when the voltage applied to the barrier is $-35 \mathrm{kV}$. 


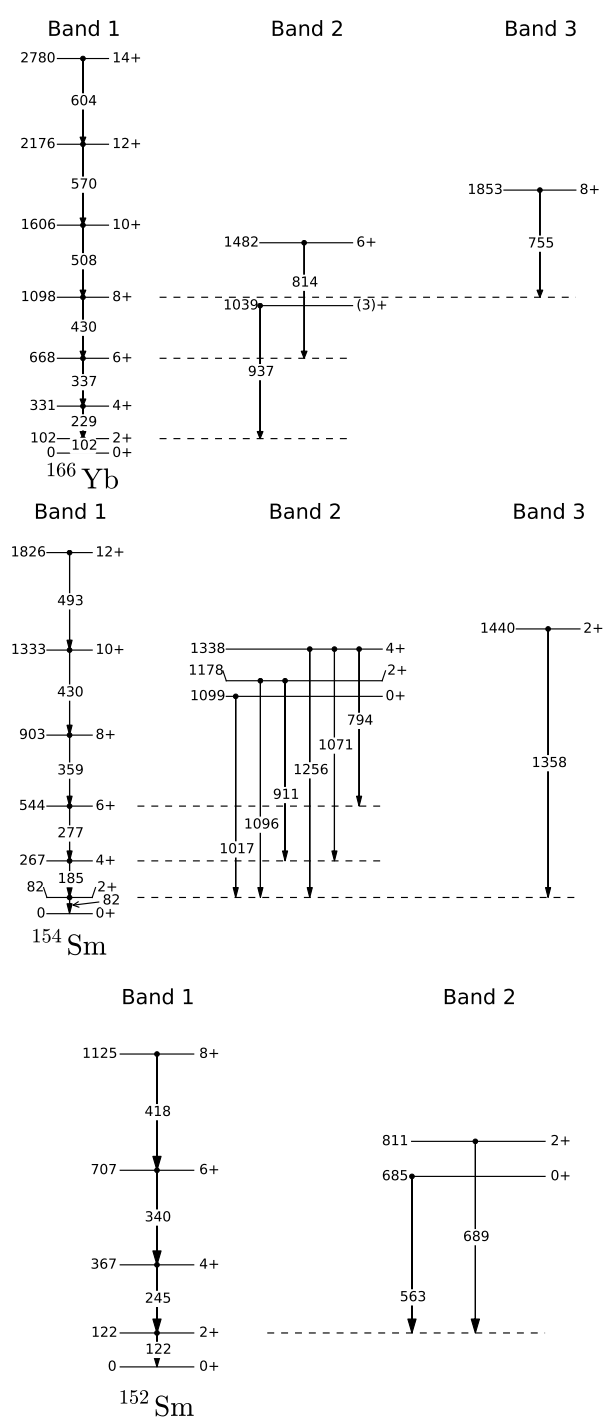

Figure 1: Partial level schemes of ${ }^{166} \mathrm{Yb},{ }^{154} \mathrm{Sm}$ and ${ }^{152} \mathrm{Sm}$. The level and transition energies are rounded to the nearest keV. Data from Ref [13].

\section{3. Data analysis procedures}

79 3.1. Electron add-back/veto

80 3.1.1. Description

${ }_{81}$ Around $1 \mathrm{MeV}$ energy the range of the electrons in silicon approaches and 82 exceeds the typical segment dimensions of the SAGE silicon detector (1 mm 


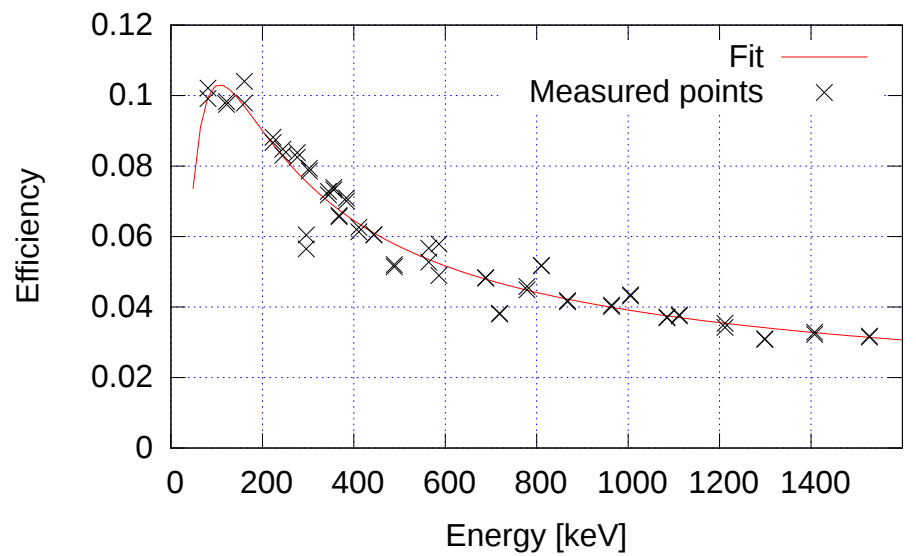

Figure 2: Detection efficiency for single $\gamma$-rays in JUROGAM II. The measured points are from calibration runs before and after the experiment. Typical errors on the measured points are $\pm 1 \%$ of the value. Error bars have been omitted for clarity.

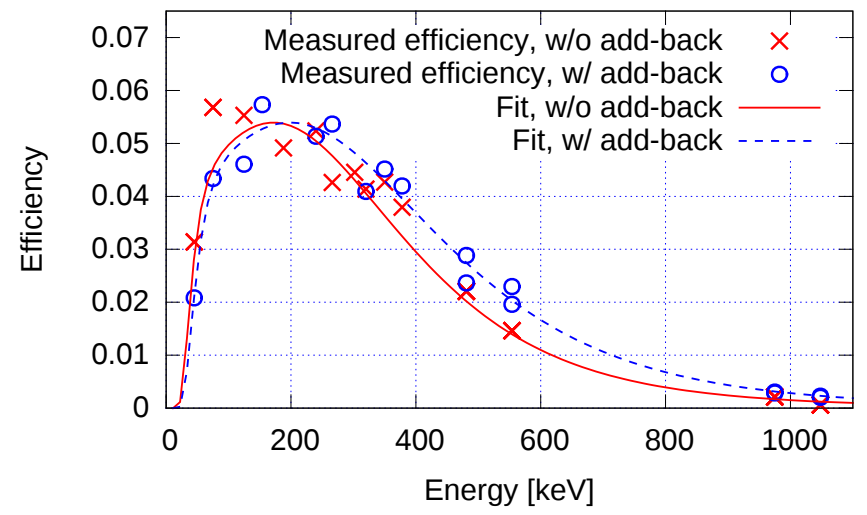

Figure 3: Absolute electron detection efficiency with and without add-back. Typical errors on the measured points are $\pm 3 \%$ of the value. Error bars are omitted for clarity.

thick, 1-2 mm wide radially). The calculated $99 \%$ stopping range for a $1 \mathrm{MeV}$ electron in silicon is $1.98 \mathrm{~mm}$. A number of the high energy electrons simply punch through the $1 \mathrm{~mm}$ thick detector and deposit only a fraction of their full 


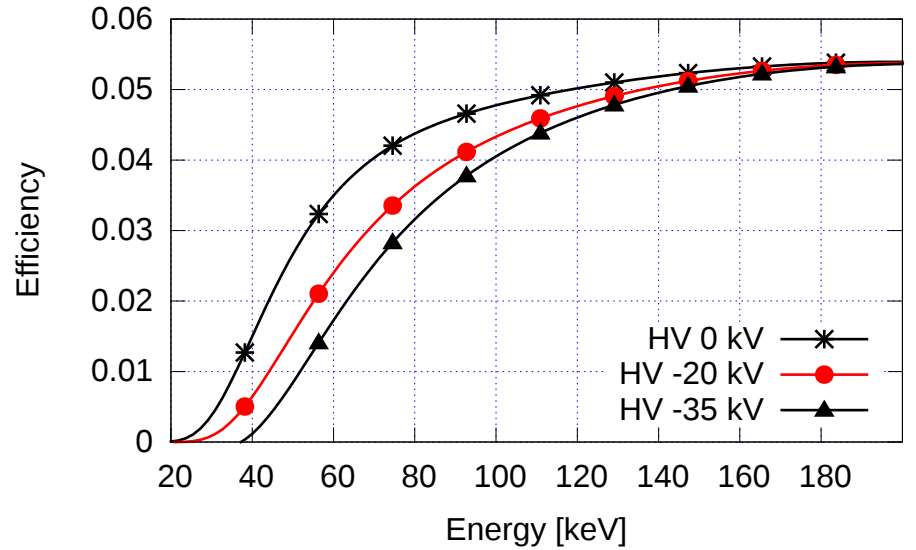

Figure 4: Effect of the HV barrier on the absolute electron detection efficiency. The lowest reliably achieved voltage in experimental conditions is $-35 \mathrm{kV}$.

energy. Moreover, a significant fraction of the high energy electrons scatter to adjacent segments and thus deposit energy in two or more segments. These events can be either recovered by summing the energies (add-back) or can be removed (vetoed) from the data with a simple algorithm. The outline of the add back/veto algorithm is shown in figure 5. An example of a typical spectrum generated by the add-back algorithm is shown in figure 6 . In the cases where two or more pixels are hit, the energy deposited in a single pixel is found to have any value up to the maximum for the transition. The inset in figure 6 shows the effect of the add-back on the resulting peak shape. Unfortunately, due to the structure of the Si-detector, some electrons lose energy in the inactive area between segments which cannot be detected and a spurious lower energy component is observed in the spectrum. Due to technical issues with bias source the silicon detector bias was limited to $90 \mathrm{~V}$ during the measurements and based on the detector I-V curves there is reason to believe that the detector is not completely depleted. This may explain the apparent high energy loss between segments shown as a difference in energy between peaks A and B in figure 6 . Note that summing events that first scatter between segments and then escape the detector would yield a continuous tail below the full energy peak and cannot 


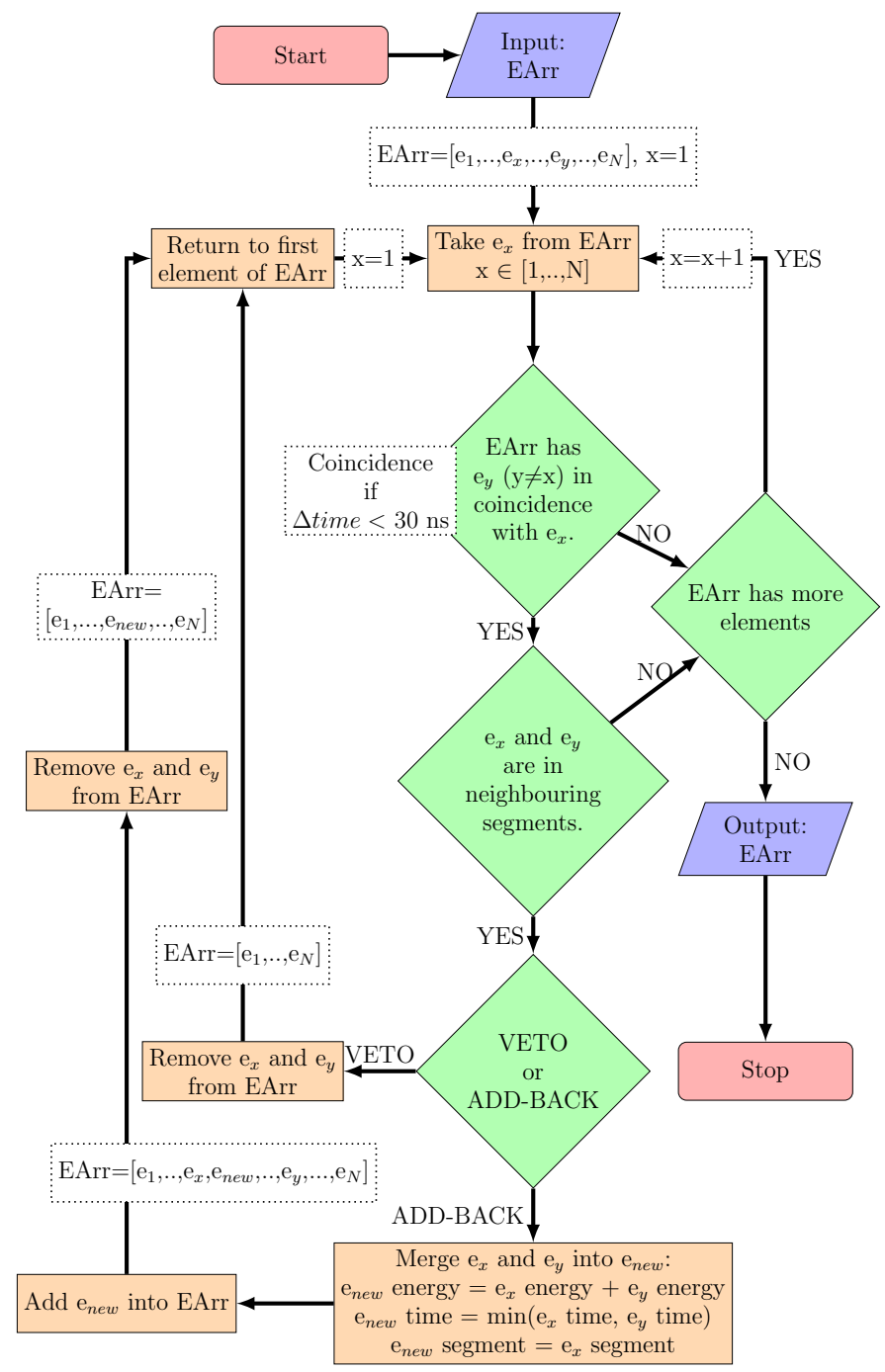

Figure 5: Outline of the add back/veto algorithm. EArr is an iterable data structure where all the elements (electrons) have energy, time and position (segment) attributes.

be the main process generating the secondary peak. Tests conducted during the commissioning phase showed that the detector does not suffer from cross talk between signal strip wires or electronics that could explain the secondary peak. High energy, "punch through" events that effect the common ground behind the silicon detector can be also ruled out as a source of the secondary peak as the 
effect in common ground dissipates over all the silicon segments.

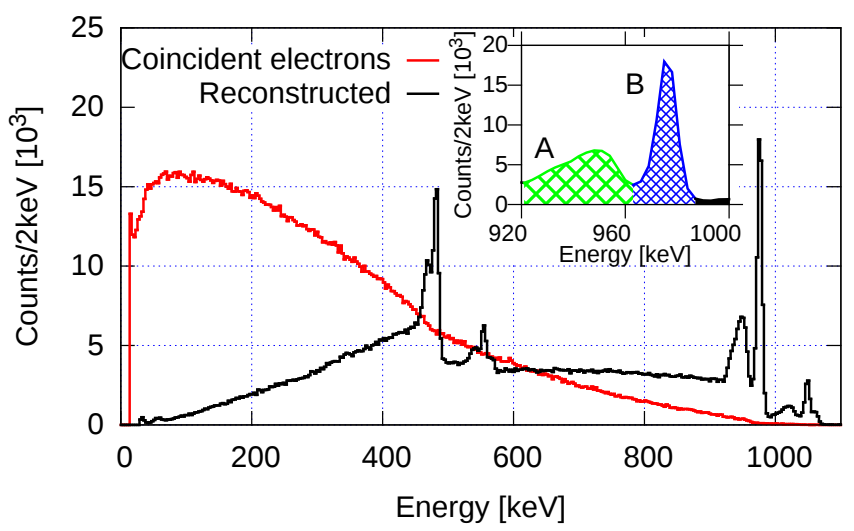

Figure 6: Conversion electron energy spectrum measured with ${ }^{207} \mathrm{Bi}$ source reconstructed by the add-back algorithm along with the original energy spectrum for events where two or more electrons are detected simultaneously in neighbouring segments. Inset A typical peak shape in the reconstructed spectrum. Peak A corresponds to electrons that are scattered through the inactive part of the detector which separates the segments. Peak B corresponds to the full energy peak.

109

\subsubsection{Neighbouring segments}

The SAGE detector is segmented in 90 individual segments and there are several different ways in which it is possible to associate the segments within the add-back procedure. A number of different schemes were tested and the optimal scheme was found to be where all the neighbouring segments within the central region of the detector are grouped together and thereafter those with the longest common borders. The emphasis in determining the best scheme was placed on maximizing the full energy peak areas while at the same time keeping the summing of full energy events with background to a minimum. An outline of the SAGE silicon detector which shows how the different segment types are searched for coincident events is illustrated in figure 7. A comparison of spectra of electrons emitted from ${ }^{207} \mathrm{Bi}$ after application of the add-back and veto algorithms is shown in figure 8 . As can be seen from figure 8(a), at energies around $550 \mathrm{keV}$ the add-back algorithm has little effect on the efficiency 


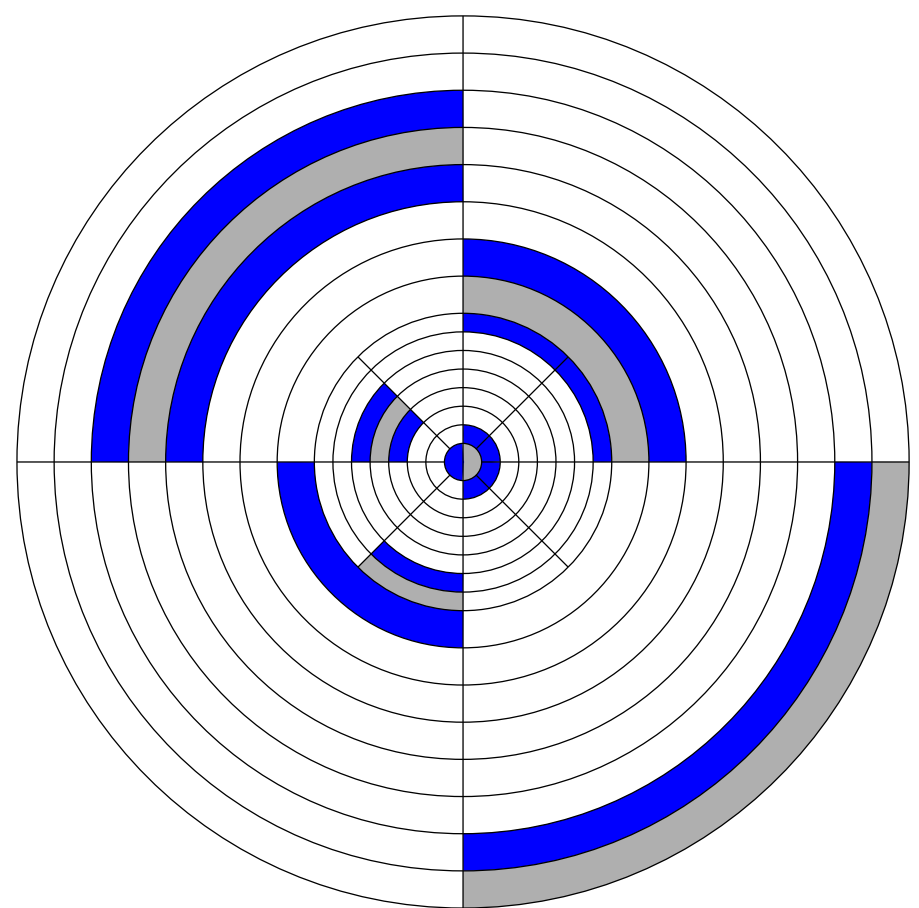

Figure 7: Add-back search patterns for six different segment types. The gray color (lighter color) shows the segment where the event with algorithm index $\mathrm{x}$ is detected (see fig. 5), the adjacent blue segments are those which are searched for coincident events.

(electron ranges are shorter) but the use of vetoing significantly reduces the background under the peaks. In figure $8(\mathrm{~b})$ it can be seen that the add-back algorithm increases the efficiency at higher energies, but the effect of vetoing is rather limited. The overall effect of the add-back procedure on the detection efficiency is shown in figure 3. The veto algorithm does not have an effect on the detection efficiency, but can be used to reduce the background in the spectrum. A similar add-back method has been devised for the SPICE electron spectrometer [14]. 

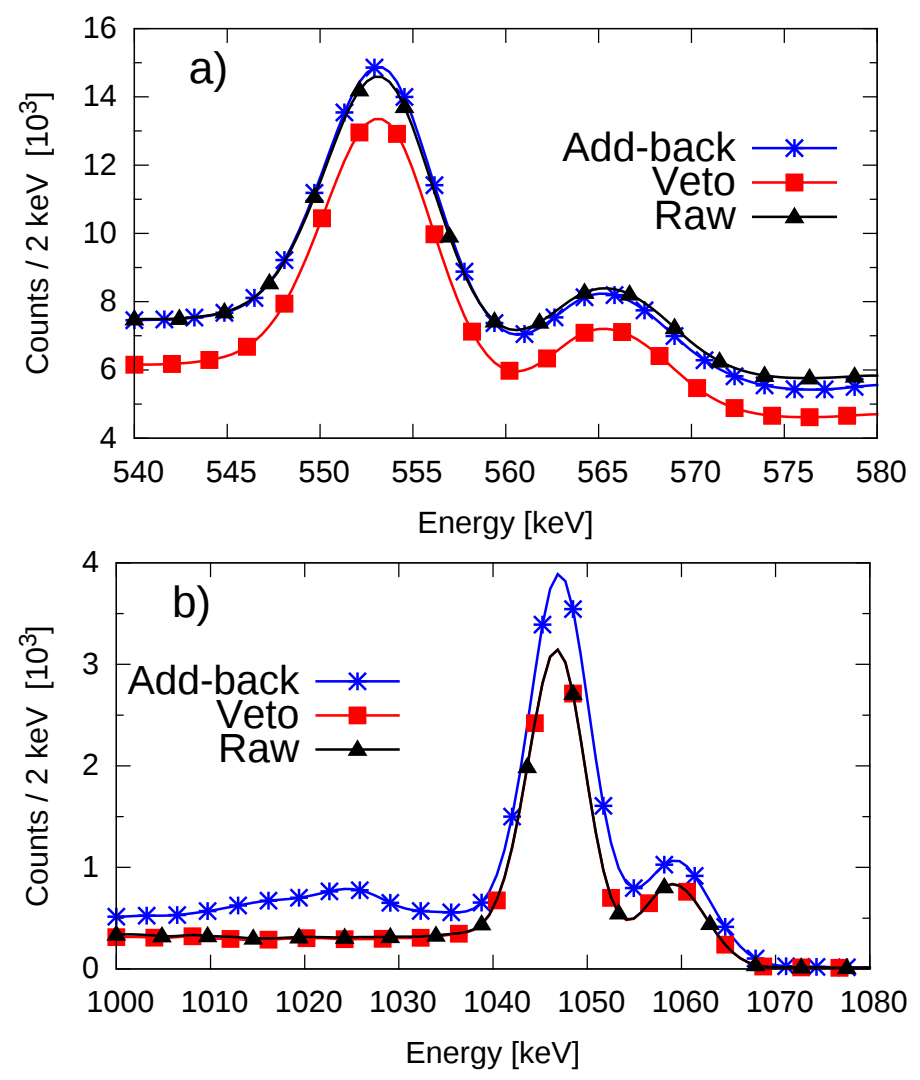

Figure 8: A comparison of the results of applying the veto and add-back algorithms on the conversion electron peaks from ${ }^{207} \mathrm{Bi}$. a) 554 and $566 \mathrm{keV}$ peaks. b) 1048 and $1060 \mathrm{keV}$ peaks. Data is smoothed to allow better differentiation between the cases.

\subsection{Filtering with detection radius}

As mentioned in the introduction, the operational principle and the design of the SAGE spectrometer gives an opportunity to filter the predominant electron background by studying the detection radius of the electrons as a function of energy. In order to develop the filter, the electron transport properties of SAGE were first probed with standard open electron sources. By using the source data the maximum allowed radius for electrons of a certain energy can be deduced.

The source data was used to determine the maximum radius as a function of energy, which was subsequently used to fit a curve based on the function for 


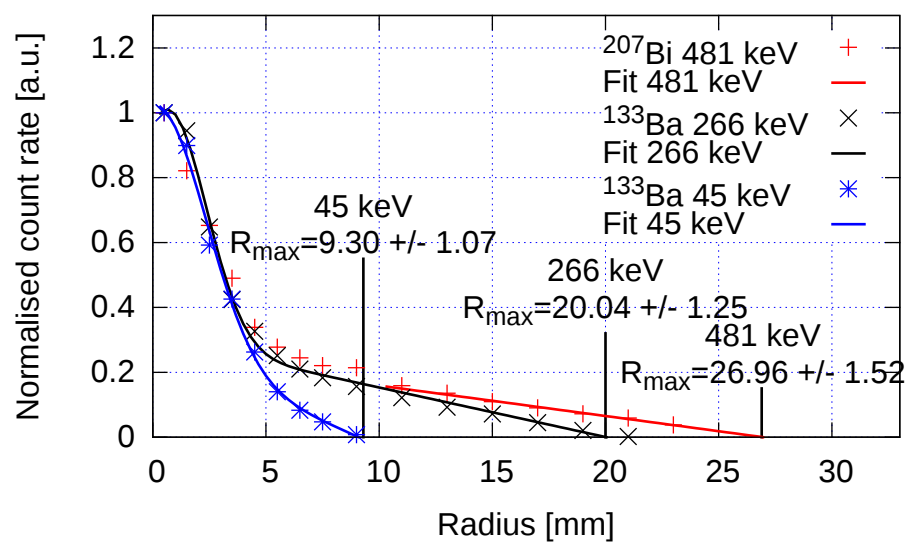

Figure 9: Distributions of electrons with various energies over the SAGE Si-detector. Fits are made according to equation 3 except that only the tail end of $481 \mathrm{keV}$ fit is shown for clarity. Note that the outer radius of the SAGE Si-detector is $24 \mathrm{~mm}$.

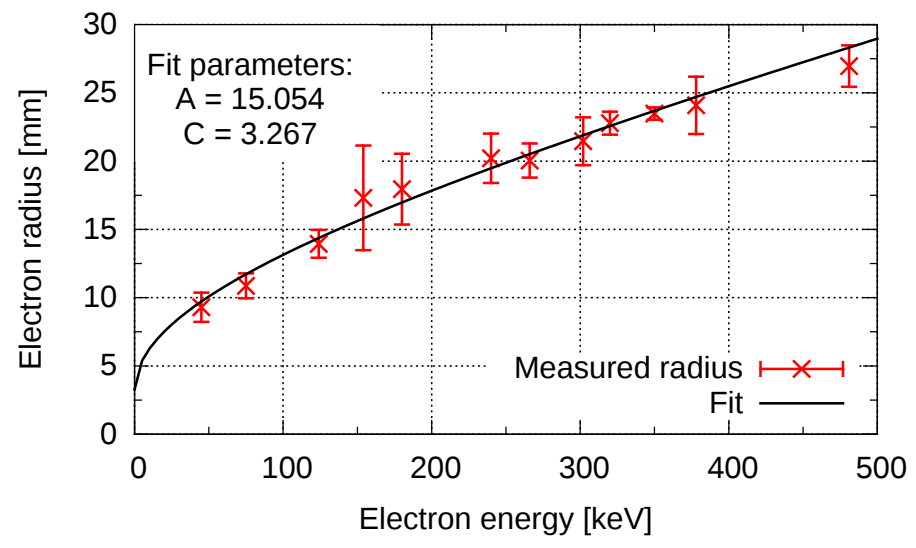

Figure 10: Measured maximum radius as a function of electron energy.

the Larmor radius. Using the relativistic form of the Larmor radius with the assumption that electron velocity is perpendicular to the magnetic field we get

$$
R_{e}(E)=\frac{\beta c \gamma m_{e}}{e B}=\frac{m_{e} c \gamma \sqrt{1-1 / \gamma^{2}}}{e B}
$$




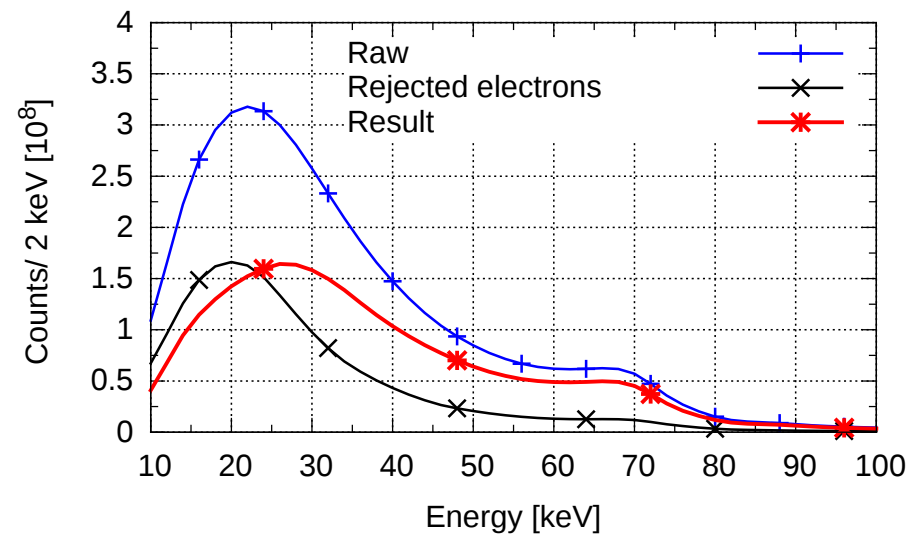

Figure 11: Effect of radial filtering on electron singles spectrum obtained from the S06 inbeam data.

$$
=\frac{m_{e} c \sqrt{\gamma^{2}-1}}{e B}=A \sqrt{\left(1+\frac{E}{m_{e} c^{2}}\right)^{2}-1}+C
$$

where $m_{e}$ is the electron mass, $\mathrm{v}$ is the velocity of the electron, $\mathrm{c}$ is the speed of light, $\beta=\mathrm{v} / \mathrm{c}, \gamma=1 / \sqrt{1-\beta^{2}}$, E is the kinetic energy of the electron, $\mathrm{e}$ is the electron charge and $\mathrm{B}$ the magnetic field.The fitting parameters are $\mathrm{A}\left(=m_{e} c / e B\right)$ and C. As the direction and the strength of the magnetic field are not clearly defined close to the SAGE silicon detector the last form of equation 2 is used as a basis for fitting. If the electron distribution is well centred electrons with energy $\mathrm{E}$ and higher radius than $\mathrm{R}_{e}(\mathrm{E})$ are assumed to have been scattered or generated by beam halo effects and considered to contribute in the background and can be filtered. An example of electron distributions with different energies is shown in figure 9. The fit function used to describe the electron distribution over the radius of the $\mathrm{Si}$-detector has the form

$$
n(r)=a \times \operatorname{Exp}\left(-b\left(r-r_{0}\right)^{2}\right)+c r+d,
$$

where $\mathrm{a}, \mathrm{b}, \mathrm{c}, \mathrm{d}$ and $r_{0}$ are fitted parameters and $\mathrm{r}$ is the radius in $\mathrm{mm}$. To avoid artefacts arising from radial segmentation of the Si-detector the electron radius is randomized within the radial segment limits. For example electron hitting one of the two center segments gets radius within range of $[0,1] \mathrm{mm}$. In an experiment, 
the position of the beam spot on the target must be carefully adjusted in order to centre the electron count rate distribution at the detector. Note that the beam spot size and the active spot size in the calibration sources is roughly the same $(\oslash \backsim 3 \mathrm{~mm})$. Originally the magnetic configuration of SAGE caused the electron distribution to systematically veer down and right from the Si-detector center (looking from the target). This was corrected by modifying magnetic shielding around the magnetic coils (see details in Ref's [3, 15]). The $\delta$-electron background is not directly filtered because it is generated in the correct position. The measured maximum radius behaviour determined using the various electron energies from ${ }^{133} \mathrm{Ba}$ and ${ }^{207} \mathrm{Bi}$ sources is shown in figure 10 along with a fit using equation 2. The filter is shown to reduce low energy background below $50 \mathrm{keV}$ by approximately $10 \%$ when using source data. The effect of radial filtering on in-beam data is much more prominent as seen in the figure 11 . The majority of the background is from $\delta$-electrons produced by interaction of the beam with the target. As in general, the current work focuses on internal conversion coefficients with transition energies higher than $50 \mathrm{keV}$ the effect on the present results is limited. Nevertheless, the filter is employed as it reduces the number of events in the $\gamma-e^{-}$coincidence matrix thus easing the analysis. Note that if the addback/veto algorithm is used in the same analysis process with radial filtering the add-back/veto must be performed first in order to avoid errors with the radial filtering arising from scattered events.

\subsection{Definition of coincidence time gates}

In order to extract accurate absolute internal conversion coefficients, the time gates for $\gamma-\gamma$ and $\gamma-e^{-}$coincidences must be carefully selected. In the present work, the $\gamma-\gamma$ and $\gamma-e^{-}$time differences were found to be energy dependent. The common practice of selecting a single time independent time gate from $\gamma-\gamma$ and $\gamma-e^{-}$time difference spectra can be lacking in this case. The correct time gate can be found by slicing the time spectra in sections and studying the relative number of coincident events within this slice compared to the total number of counts in the coincident peak. As an example, relative peak curves 
from ${ }^{154} \mathrm{Sm} 82-185 \mathrm{keV}, 185-277 \mathrm{keV},{ }^{166} \mathrm{Yb} 102-430 \mathrm{keV}$ and random ${ }^{154} \mathrm{Sm}$ $82-{ }^{152} \mathrm{Sm} 122 \mathrm{keV}$ coincidences are presented in figure 12 . The time gate is defined to be the time interval where the relative coincidence peak area is larger than random peak area. As an example the ${ }^{154} \mathrm{Sm} 82-185 \mathrm{keV}$ coincidence gives a high limit of 100ns and a low limit of -60ns (points are circled in figure 13). Due to the experimental timing logic the low limit is related to de-excitation observed first and the high limit to the second in the coincidence cascade in the $\gamma-\gamma$ data. Timing of electrons is less affected by electron energy and the gate limits can be set as a function of $\gamma$-ray energy with $\gamma-e^{-}$data. Time gates defined from ${ }^{133} \mathrm{Ba},{ }^{207} \mathrm{Bi},{ }^{154} \mathrm{Sm}$ and ${ }^{166} \mathrm{Yb}$ data are shown in figures 13 and 14. The fits presented in figures 13 and 14 have a general form of

$$
f(E)=A 1 \times \operatorname{Exp}\left(\frac{B 1}{\sqrt{E}}\right)+C 1,
$$

where $\mathrm{E}$ is the energy in $\mathrm{keV}$ and $\mathrm{A} 1, \mathrm{~B} 1, \mathrm{C} 1$ are the fitted parameters.

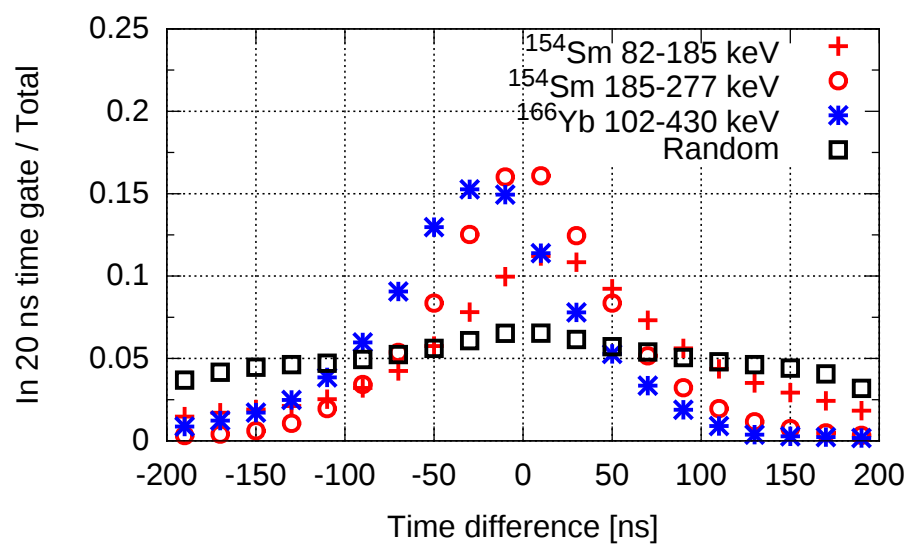

Figure 12: Relative coincident peak size curves compared to total number of coincident counts within [-200:200]ns time gate. 


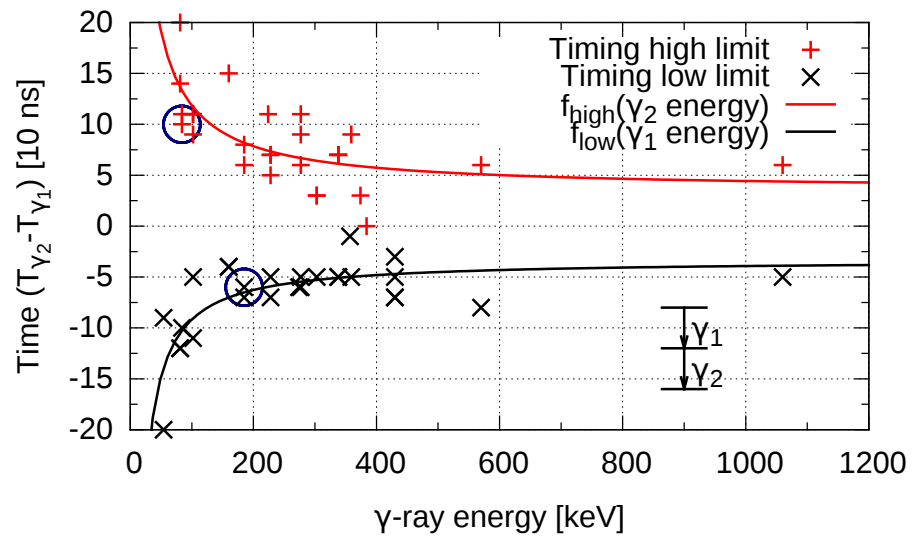

Figure 13: Energy-dependent time gate limits for $\gamma-\gamma$ coincidences.

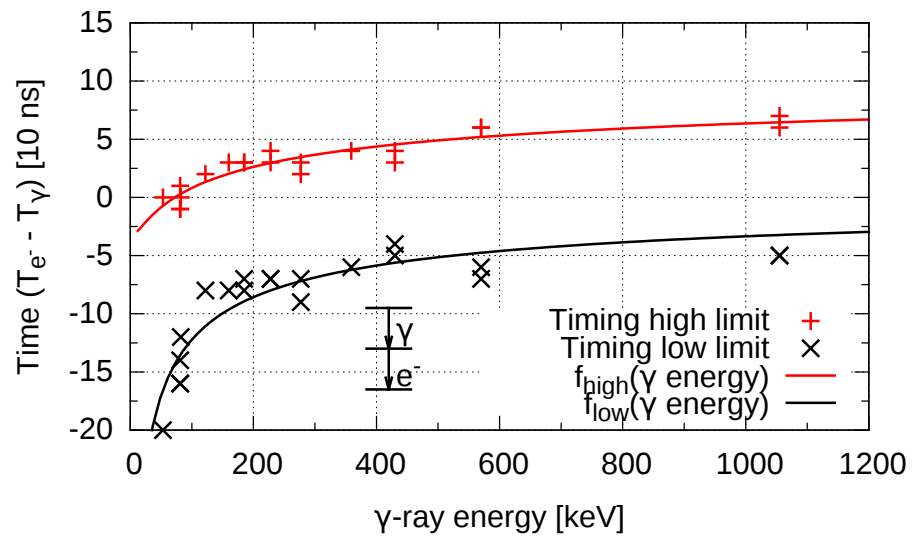

Figure 14: Energy-dependent time gate limits for $\gamma-e^{-}$coincidences. Both limit fits are functions of $\gamma$-ray energy.

\section{Results}

4.1. Extraction of internal conversion coefficients

In this work the internal conversion coefficient $\alpha_{\text {exp }}$ is determined from

$$
\alpha_{e x p}=\frac{N_{e} \times \epsilon_{\gamma}}{N_{\gamma} \times \epsilon_{e}},
$$

where $\mathrm{N}_{e}$ is the number of detected electrons, $\mathrm{N}_{\gamma}$ is the number of detected $\gamma$-rays, $\epsilon_{\gamma}$ and $\epsilon_{e}$ are the detection efficiencies for $\gamma$-rays and electrons, respectively. In this work, the effect of angular correlations is neglected. Using the 
data obtained in the S06 experiment, a large number of experimental internal conversion coefficients could be determined. The measured absolute internal conversion coefficients for ${ }^{154} \mathrm{Sm},{ }^{152} \mathrm{Sm}$ and ${ }^{166} \mathrm{Yb}$ ground state band transitions that are all considered to be pure E2 character as a function of electron energy are shown in figure 15. The results derived from the raw (no algorithms applied and unfiltered) matrices differ from the reference tabulated values obtained using BrIcc [16] conversion coefficient calculator. It can be seen that no single normalisation constant would yield agreement throughout the full energy range and without a common factor the NPG method cannot be used. After application of time gates, either add-back or veto and radial filtering for the electron events in the silicon detector the overall result is much more agreeable. A more detailed plot of the final result is shown in figure 16. As can be seen in figure 16 the measured ICCs below $200 \mathrm{keV}$ are systematically lower than the tabulated values. The difference is thought to be the result of the interactions of the electrons with the thick target. The values deduced indicate that ${ }^{166} \mathrm{Yb}$ is less affected. The difference can arise from the fact that ${ }^{166} \mathrm{Yb}$ is produced in a fusion-evaporation reaction, meaning that ${ }^{166} \mathrm{Yb}$ has a kinetic energy of only $\backsim 6 \mathrm{MeV}$ and range in samarium of $\backsim 0.9 \mathrm{mg} / \mathrm{cm}^{2}$ with beam $\left({ }^{16} \mathrm{O}\right)$ energy of $65 \mathrm{MeV}$. This should be compared with that for excited ${ }^{154} \mathrm{Sm}$ which is $\backsim 4$ $\mathrm{mg} / \mathrm{cm}^{2}$. As the beam particles pass through the target matter they lose energy and therefore creation of sub-barrier fusion products deeper in the target matter is less likely. As the electrons emitted from ${ }^{166} \mathrm{Yb}$ travel through less target matter the energy loss is smaller and probability of scattering is lower hence it is more likely that the emitted electrons contribute to the full energy peaks. According to the rule-of-thumb given in Ref [17] the optimal target thickness for measurements of conversion electrons in an energy range of 100 to $500 \mathrm{keV}$ would be $0.3-0.7 \mathrm{mg} / \mathrm{cm}^{2}$. This is significantly less than the $1.5 \mathrm{mg} / \mathrm{cm}^{2}$ target used in this case and negative effects on the spectrum quality can be expected. The relative effect of the target on electron transmission is shown figure 17. If we process the ICCs measured below $200 \mathrm{keV}$ with the rough assumption that samarium conversion electrons originate evenly throughout the target depth 


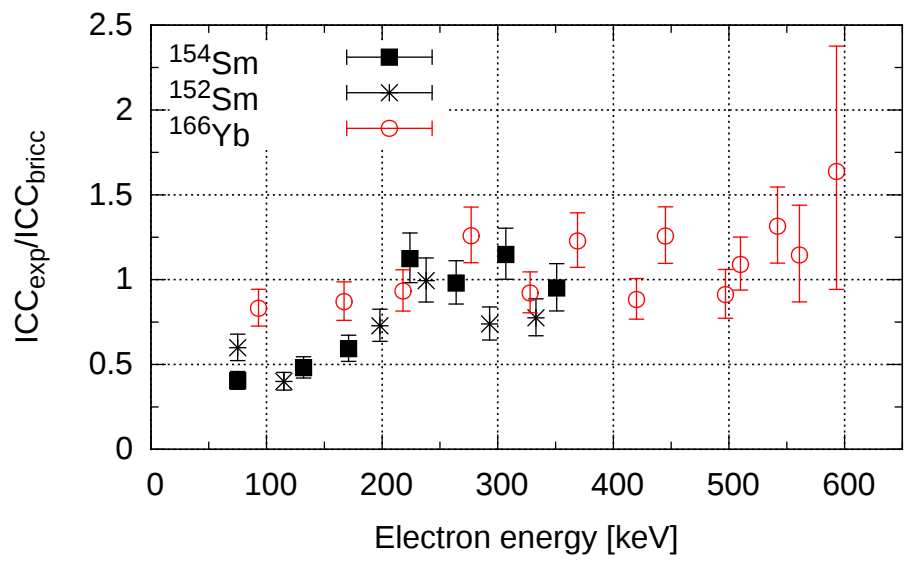

Figure 16: Comparison of the experimental ICCs relative to tabulated values for isotopes of Samarium and ${ }^{166} \mathrm{Yb}$ obtained with add-back algorithm. The better agreement for ${ }^{166} \mathrm{Yb}$ below $200 \mathrm{keV}$ can be understood in terms of the reaction kinematics and the interactions of electrons with the target.

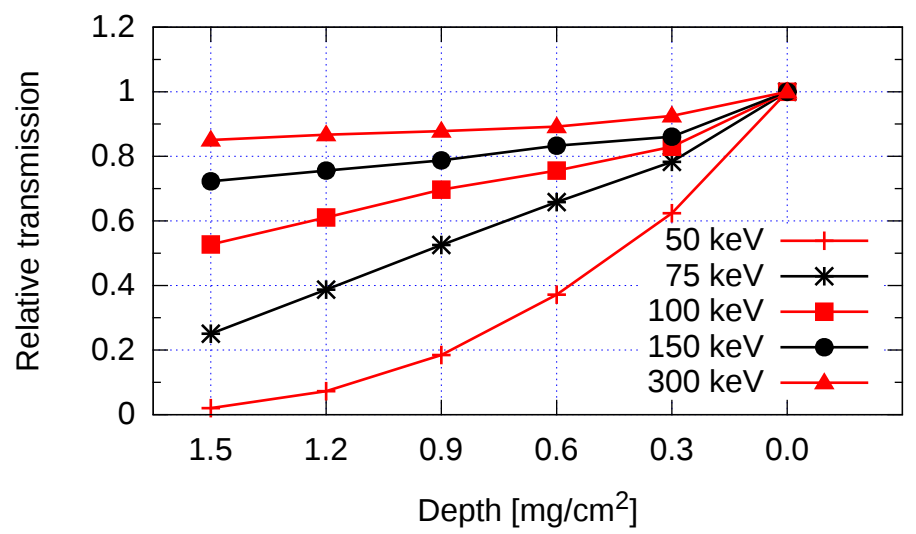

Figure 17: Effect of Sm target on electron transmission. Simulation result taking account of energy loss, scattering and SAGE electron acceptance.

the energy dependent gates shown in figures 13 and 14 are significantly different. If we consider the energy range between $200-400 \mathrm{keV}$ the $\gamma-\gamma$ time independent gate is roughly half the width of the energy dependent time gate. According to equation 5 this would yield ICCs approximately a factor of 2 too large as $\mathrm{N}_{\gamma}$ is halved. This would explain why the measured ICCs obtained in Ref [11] were 
Table 2: Approximate attenuation coefficients for ICCs below $200 \mathrm{keV}$ and resulting relative ICCs with results obtained with add-back algorithm.

\begin{tabular}{|c|c|c|c|c|}
\hline Origin & $\begin{array}{c}e^{-} \text {energy } \\
{[\mathrm{keV}]}\end{array}$ & $\alpha_{\text {Rel }}{ }^{a}$ & $\begin{array}{c}\text { Attenuation } \\
\text { coefficient }^{b}\end{array}$ & $\begin{array}{c}\text { Corrected } \\
\alpha_{\text {Rel }}\end{array}$ \\
\hline \hline${ }^{152} \mathrm{Sm}$ & 75 & $0.59(7)$ & $0.6(1)$ & $1.0(2)$ \\
& 115 & $0.39(5)$ & $0.7(1)$ & $0.6(4)$ \\
& 198 & $0.73(9)$ & $0.8(1)$ & $0.9(2)$ \\
\hline${ }^{154} \mathrm{Sm}$ & 75 & $0.41(5)$ & $0.6(1)$ & $0.7(3)$ \\
& 132 & $0.48(6)$ & $0.7(1)$ & $0.7(3)$ \\
& 171 & $0.59(7)$ & $0.8(1)$ & $0.7(3)$ \\
\hline${ }^{166} \mathrm{Yb}$ & 93 & $0.83(9)$ & $0.8(1)$ & $1.0(2)$ \\
& 167 & $0.87(9)$ & $0.9(1)$ & $1.0(2)$ \\
\hline
\end{tabular}

${ }^{a} \alpha_{\text {Rel }}=\alpha_{\text {exp }} / \alpha_{B r I c c}$

${ }^{b}$ Approximated from figure 17

1.8 times the literature value ${ }^{2}$.

\subsection{Internal conversion coefficients of high-energy transitions}

The main goal of the S06 ${ }^{154} \mathrm{Sm}$ Coulomb excitation experiment was to study inter-band transitions between the excited side bands and the ground state band. The results for these higher energy transitions are shown in table 3. In several cases only an upper limit could be given due to the lack of statistics. The partial level schemes showing the transitions investigated are shown in figure 1. Data for level schemes are from Ref [13]. The measured ICC for the $4_{3}^{+}$to $6_{1}^{+}$ transition in ${ }^{154} \mathrm{Sm}$ suggests E2 character. However, a M1+E2 transition with a mixing ratio $\delta \lesssim 0.5$ is possible within the error limits.

\footnotetext{
${ }^{2}$ J. Smallcombe, private communication
} 
Table 3: Experimental results for $\alpha_{K}$ ICCs in ${ }^{154} \mathrm{Sm}$, obtained by using energy dependant time gates, either the veto or add-back algorithm and filtering. The level and multipolarity assignments are as listed in NNDC [13] if not otherwise stated. The upper limits are deduced with a $90 \%$ confidence limit according to Ref [18]. The measured value of the mixing ratio $(\delta)$ is given where available.

\begin{tabular}{|c|c|c|c|c|c|c|c|c|c|}
\hline Origin & $\begin{array}{c}\gamma \text { energy } \\
{[\mathrm{keV}]}\end{array}$ & $\begin{array}{c}e^{-} \text {energy } \\
{[\mathrm{keV}]}\end{array}$ & $I_{i}^{\pi}$ & $I_{f}^{\pi}$ & $\sigma \mathrm{L}$ & $\delta$ & $\begin{array}{c}\alpha_{K, \text { exp }} \\
\text { add-back }\end{array}$ & $\begin{array}{c}\alpha_{K, \text { exp }} \\
\text { veto }\end{array}$ & $\alpha_{K, \text { lit }}$ \\
\hline \hline${ }^{152} \mathrm{Sm}$ & 563 & 516 & $0_{2}^{+}$ & $2_{1}^{+}$ & $\mathrm{E} 2$ & - & $0.006(4)$ & $0.005(3)$ & $0.0069(34)^{a}$ \\
& 689 & 642 & $2_{2}^{+}$ & $2_{1}^{+}$ & $\mathrm{E} 0+\mathrm{M} 1+\mathrm{E} 2$ & $8_{-3}^{+6^{d}}$ & $0.003(2)$ & $0.04(2)$ & $0.0297(75)^{a}$ \\
\hline${ }^{154} \mathrm{Sm}$ & 795 & 748 & $4_{3}^{+}$ & $6_{1}^{+}$ & $(\mathrm{E} 2)^{b}$ & - & $0.0030(27)$ & $\leq 0.021(1)$ & $0.00345(5)^{c}$ \\
& 911 & 864 & $2_{3}^{+}$ & $4_{1}^{+}$ & $\mathrm{E} 2$ & - & $0.0023(20)$ & $0.003(2)$ & $0.0034(16)^{a}$ \\
& 1017 & 970 & $0_{3}^{+}$ & $2_{1}^{+}$ & $\mathrm{E} 2$ & - & $\leq 0.038(1)$ & $\leq 0.018(1)$ & $0.00204(3)^{c}$ \\
& 1071 & 1024 & $4_{3}^{+}$ & $4_{1}^{+}$ & $\mathrm{M} 1+\mathrm{E} 2$ & $>50^{e}$ & $\leq 0.025(1)$ & $\leq 0.038(1)$ & $0.0079_{-0.0073}^{+0.0087}{ }^{a}$ \\
& 1096 & 1050 & $2_{3}^{+}$ & $2_{1}^{+}$ & $\mathrm{M} 1+\mathrm{E} 2$ & $30(21)^{f}$ & $\leq 0.036(1)$ & $\leq 0.043(1)$ & $\leq 0.0067(6)^{a}$ \\
& 1256 & 1209 & $4_{3}^{+}$ & $2_{1}^{+}$ & $\mathrm{E} 2$ & - & $\leq 0.061(1)$ & $\leq 0.051(2)$ & $0.001329(19)^{c}$ \\
& 1358 & 1311 & $2_{4}^{+}$ & $2_{1}^{+}$ & {$[\mathrm{M} 1+\mathrm{E} 2]$} & $19(10)^{f}$ & $\leq 0.036(1)$ & $\leq 0.032(1)$ & $0.0014(3)^{c}$ \\
\hline${ }^{166} \mathrm{Yb}$ & 755 & 694 & $8_{3}^{+}$ & $8_{1}^{+}$ & $\mathrm{E} 0+\mathrm{M} 1+\mathrm{E} 2$ & - & $0.03(2)$ & $0.005(4)$ & $0.0158(45)^{a}$ \\
& 814 & 753 & $6_{2}^{+}$ & $6_{1}^{+}$ & $\mathrm{M} 1$ & - & $0.008(3)$ & $0.008(3)$ & $0.0069(28)^{a}$ \\
& 937 & 876 & $(3)_{2}^{+}$ & $2_{1}^{+}$ & $\mathrm{E} 2$ & - & $0.014(6)$ & $\leq 0.49(2)$ & $0.00351(5)^{c}$ \\
\hline
\end{tabular}

${ }^{a}$ Experimental result from Ref [11].

${ }^{b}$ Not available in NNDC, own assignment based on the experimental ICC value.

${ }^{c}$ BrIcc result. ${ }^{d}$ From Ref [19]. ${ }^{e}$ From Ref [13]. ${ }^{f}$ From Ref [20].

\subsection{Observation of high-energy events in the silicon detector}

As can be seen in figure 18, the electron spectrum measured from the ${ }^{16} \mathrm{O}+{ }^{154} \mathrm{Sm}$ Coulomb excitation reaction shows a significant number of events that lie outside the dynamic range of the analogue to digital converters in the data acquisition system. The number of these events far exceeds that which is expected based on the behaviour and shape of the electron spectrum at high energies. In a test experiment (ST1), these "overflow" events were shown to be due to the detection of backscattered ${ }^{16} \mathrm{O}$ beam in the SAGE silicon detector. 


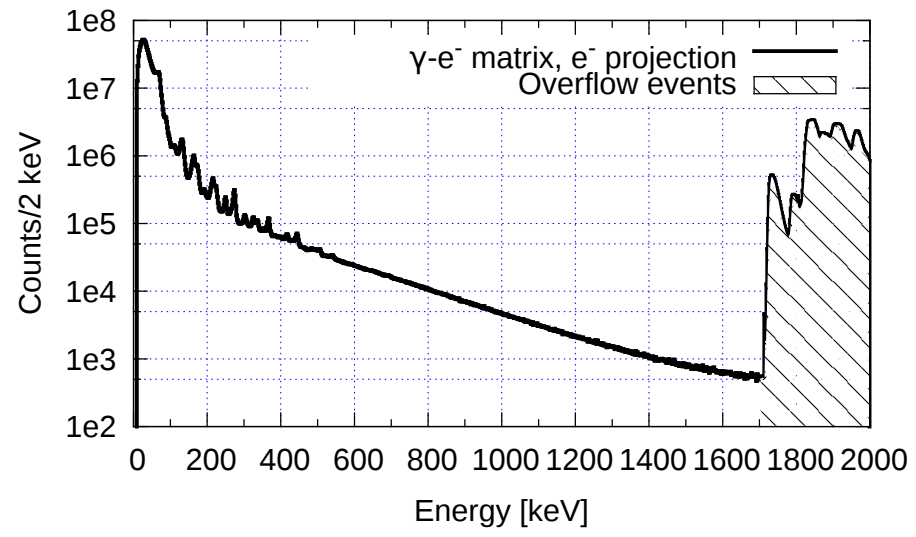

Figure 18: A projection of the electron spectrum from the $\gamma$-electron coincidence matrix produced in the ${ }^{16} \mathrm{O}+{ }^{154} \mathrm{Sm}$ Coulomb excitation reaction. Note the abundance of "overflow" events observed at high energies.

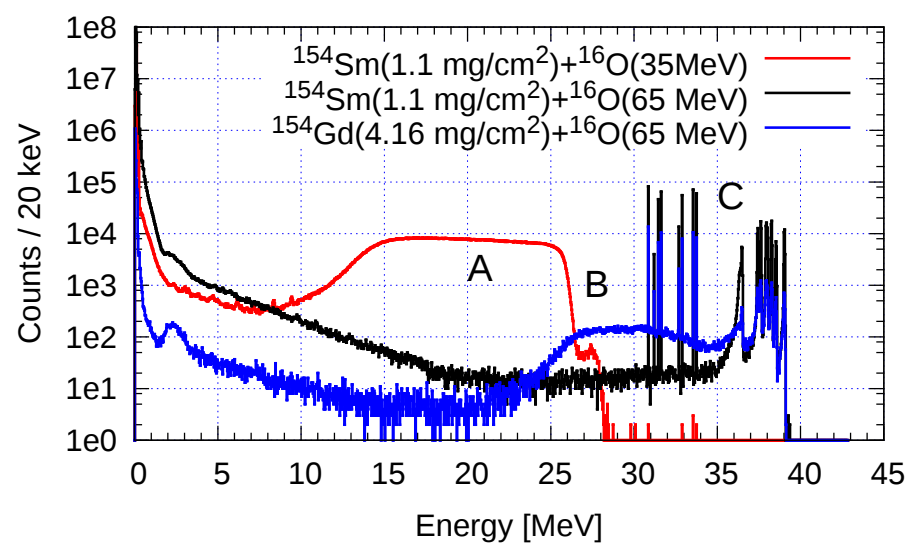

Figure 19: Spectra from the SAGE silicon detector with increased dynamic range from the ST1 test run with a ${ }^{154} \mathrm{Sm}$ target bombarded by a beam of ${ }^{16} \mathrm{O}$ at an energy of $35 \mathrm{MeV}$ and $65 \mathrm{MeV}$ and ${ }^{154} \mathrm{Gd}$ target bombarded with $65 \mathrm{MeV}$ energy. (A) Backscattered ${ }^{16} \mathrm{O}$ from samarium. (B) Backscattered ${ }^{16} \mathrm{O}$ from gadolinium. (C) Overflow events.

In the ST1 test experiment, the gain in amplification of the outer segment (59 to 90) signals of the SAGE silicon detector were reduced by voltage dividers allowing increased dynamic range and detection of backscattered ${ }^{16} \mathrm{O}$ beam particles. An alpha source was used for energy calibration confirming that the maximum energy range had increased to $\backsim 30-40 \mathrm{MeV}$ from the orig- 


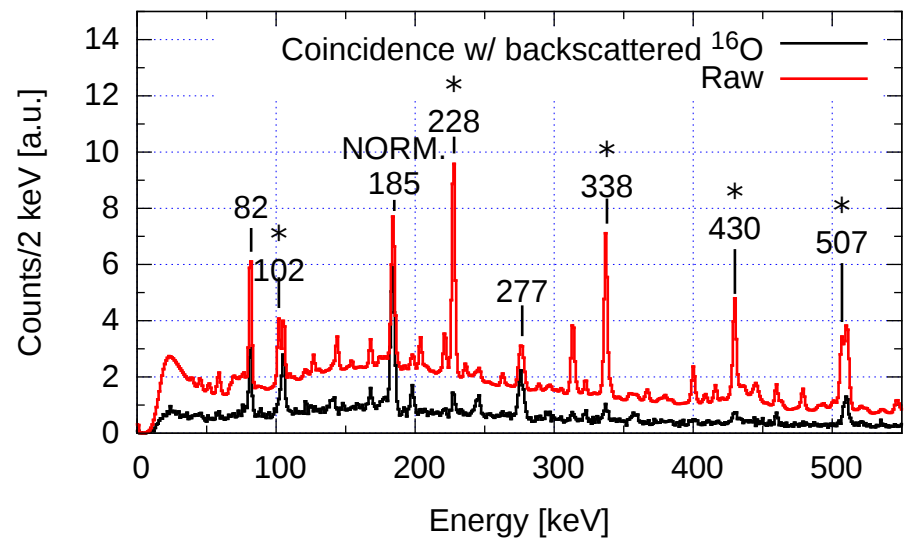

Figure 20: Gamma-projections from $\gamma$-electron matrices. Normalized with the area of 185 keV peak. From ST1 test run with $65 \mathrm{MeV}$ beam energy. Peaks marked with asterisk originate from ${ }^{166} \mathrm{Yb}$.

inal $\backsim 2-2.5 \mathrm{MeV}$. The original Coulomb excitation experiment was repeated with beam energies of 35 and $65 \mathrm{MeV}$. For validation purposes scattering from $4.16 \mathrm{mg} / \mathrm{cm}^{2}{ }^{154} \mathrm{Gd}$ target with $65 \mathrm{MeV}$ beam energy was also studied. In the resulting spectrum shown in figure 19 backscattered ${ }^{16} \mathrm{O}$ is clearly observable. The energy distribution of the scattered particles correspond with calculated distribution from a $1.1 \mathrm{mg} / \mathrm{cm}^{2}{ }^{154} \mathrm{Sm}$ target. The identification of backscattered ${ }^{16} \mathrm{O}$ was further confirmed when the beam energy was changed, resulting in a corresponding shift in the energy of the backscattered particles. Selecting events in coincidence with backscattered ${ }^{16} \mathrm{O}$, the events arising from sub-barrier fusion such as ${ }^{166} \mathrm{Yb}$ can be removed. A normalized $\gamma$-ray projection coincident with backscattered ${ }^{16} \mathrm{O}$ ions is shown in figure 20. After demanding the coincidence, the contribution from ${ }^{166} \mathrm{Yb}$ (102, 228, 338, 430 and $507 \mathrm{keV}$ peaks) is significantly smaller compared to that from ${ }^{154} \mathrm{Sm}(82,185$ and $277 \mathrm{keV})$. However, the peak areas are significantly reduced. In the raw projection of the $\gamma-e^{-}$matrix the peak area of the $185 \mathrm{keV}$ transition is in the order of $10^{8}$ but in the gated projection only on the order of $10^{3}$. Since the statistics obtained in the short test run were rather low, the data were not analysed further. However, with a longer run and improved detection system demanding a coincidence with 
scattered particles could produce exceptionally clean data for the extraction of ICCs.

\section{Conclusions}

Experimental internal conversion coefficients have been successfully extracted with non-reference based methods. The crucial step in order to determine absolute ICC values with reasonable accuracy is the creation of energy-dependent time gates. The result obtained through demanding coincidence with backscattered ions (Fig 20) resembles greatly the results obtained with recoil gating or recoil-decay tagging in $\gamma$-ray spectroscopic studies (see for example Ref [21]). In order to fully exploit the possibilities of this method plans to instrument SAGE with an additional heavy ion detector have been made. One notable detector based on recent developments with optical fibres such as presented in Ref [22] is under consideration.

\section{Acknowledgements}

This work has been supported through the UK Science and Technology Facilities Council, the Academy of Finland under the Finnish Centre of Excellence Programme 2006-2011 (Nuclear and Accelerator Based Physics Contract No. 213503), and the European Research Council under the SHESTRUCT project

(Grant Agreement No. 203481). The support from GAMMAPOOL network is acknowledged. 
[1] P. Nolan, Annu. Rev. Nucl. Part.Sci. 45 (1994) 561-607.

[2] P. Papadakis, University of Liverpool, PhD Thesis (2010, Accessed 201506-17). https://www.liv.ac.uk/physics/research/nuclear-physics/ projects/sage/publications/.

[3] J. Sorri, Phys. Scr. 85 (2012) 055201.

[4] J. Pakarinen, EPJ A (2014) 50-53.

[5] M. Leino, NIM B 99 (1995) 653.

[6] J. Sarén, NIM A 654 (2011) 508 - 521.

[7] R. Page, NIM B 204 (2003) 634.

[8] K.-H. Schmidt, et al., Phys. Lett. B 168 (1986) 39.

[9] M. Scheck, Phys. Rev. C 83 (2011) 037303.

[10] A. Mistry, University of Liverpool, PhD Thesis (2014, Accessed 2015-0617). http://repository.liv.ac.uk/.

[11] J. Smallcombe, Phys. Lett. B 732 (2014) 161-166.

[12] J. Hamilton, Nuclear Data Sheets. Section A 1 (1965) $521-602$.

[13] B. N. L. National Nuclear Data Center, Nudat2 database (2015, Accessed: 2015-05-22). http://www.nndc. bnl.gov/nudat2/.

[14] S. Ketelhut, NIM A 753 (2014) $154-163$.

[15] D. M. Cox, Eur. Phys. J. A 51 (2015) 64.

[16] T. Kibédi, NIM A 589 (2008) 202 - 229.

[17] J. Dionisio, NIM A 414 (1998) 239 - 260.

[18] L. A. Currie, Analytical Chemistry 40 (1968) 586-593. 
332 [19] K. Krane, ATOMIC DATA AND NUCLEAR DATA TABLES 16 (1975)

$333 \quad 383-408$.

334 [20] Möller, Phys. Rev. C 86 (2012) 031305.

335 [21] J. Uusitalo, NIM B 204 (2003) $638-643$.

336 [22] P. Finocchiaro, Nuclear Physics News 24 (2014) 34-39. 